\title{
A Case of Aleukemic Myelosis under the Clinical Guise of Aplastic Anemia.
}

43rd Hematological Paper.

$\mathrm{By}$

\section{Shizuo Kimura.}

(木 村 静 雄)

(From the Department of Pediatrics, Faculty of Medicine, Tohoku Imperial University, Sendai.

Director: Prof. A. Sato.)

\section{Introduction.}

Aplastic anemia is usually regarded as a well-defined, readily recognizable clinical entity. But among many cases clinically diagnosed as aplastic anemia or panmyelophthisis, there have indubitably really been examples of aleukemic lymphadenosis or aleukemic myelosis, generally with a leucopenic blood picture.

In the present paper I shall report a case which was clinically believed as a case of aplastic anemia or rather one of panmyelophthisis, but was proved by the subsequent post-mortem examination to be one of leucopenic aleukemic (subleukemic) myelosis, or one of myeloid leukemia with a decrease instead of an increase in the total number of leucocytes.

The term aleukemic myelosis is usually used to describe the following conditions (Naegeli, ${ }^{1)}$ Piney, ${ }^{2)}$ Schilling, ${ }^{3)}$ and Whitby and Britton ${ }^{4}$ ): A morbid condition in which the histological changes in the blood-forming organs are characteristic of a myelosis (leukemic infiltrations of many organs), yet the total white cell count of the peripheral blood is within normal limits or even leucopenic (subleukemic and 533 .

1) O. Na ge li, Blutkrankheiten und Blutdiagnostik, 5th edit., Berlin 1931, 453

2) A. P iney, Diseases of the blood, and edit., London 1932, 108.

3) V. Schilling, Das Blutbild, 9th and 10th edit., Jena 1933, 235. 1937,404 .

4) L. E. H. Whitby and C.J.C. Britton, Disorders of the blood, 2nd edit, 
leukemia). There is the typical qualitative alteration of blood picture, and occasionally the picture is even almost normal, with the difference that only a very few myelocytes are present. The spleen is enlarged as seen in a leukemic case, but there is no enlargement of lymph nodes. Quite often an aleukemic leukemia terminates as a frank leukemia ; the reverse can also occur, but less frequently.

Henschen and Jezler ${ }^{5)}$ examined a case of aleukemic myelosis under the feature of panmyelophthisis, and divided the clinical pictures according to the lesions of the bone marrow into 2 groups :a. Hypo- and aleukemic myelosis with 'functional' (often pure 'virtual') panmyelophthisis (better panmyeloparesis or insufficiency of discharge*), the bone marrow presenting that in the case of typical leukemia ; b. Hypo- and aleukemic myelosis with aplastic bone marrow; in this latter case the bone marrow has become aplastic under the influence of the treatment with irradiation or benzene or through functional exhaustion (real panmyelophthisis).

Several cases of aleukemic myelosis with blood picture of aplastic anemia or panmyelophthisis which terminate in evidently typical leukemia were reported (see later). My own case might have remained with diagnosis of aplastic anemia but for the post-mortem examination.

\section{The Present Case.}

The patient S.H., a boy aged $33 / 4$ years, was admitted to hospital on November 19,1934, with a month's history of progressive weakness and pallor.

The history was that he was fed on his mother's milk in his infancy and had not suffered from measles and whooping cough. $\mathrm{He}$ had never excreted any parasite and received no medical treatment which might cause a blood disease-agranulocytotic disease, for instance. His family history was of no consequence.

Two months prior to the admission, the fever had suddenly risen to $39^{\circ} \mathrm{C}$ with a temporary hematuria two or three times, but the temperature fell to normal on the next morning without any medical treatment. Such a temporary fever attacked him again during an evening one month later, and the next morning his family noticed the remarkable pallor of the patient's face. Since that day, the weakness

5) C. Hensehen and A. Jezler, Zschr. f. klin. Med, 1935, 128, 343.

* Loslösungsinsuffiziens in the original. 
and the pallor were always progressive, and the appetite became worse. and worse. Stool was once a day and had yellowish-brown colour. There was abdominal pain which would disappear after every defecation. The abdomen had, the parents stated, been distended since infancy so the family did not consider it as abnormal. Before the admission there were several nasal bleedings which would not be stopped easily.

On admission to Hospital, his temperature was $38^{\circ} \mathrm{C}$, and it was always between $37^{\circ} \mathrm{C} \sim 38^{\circ} \mathrm{C}$ throughout the entire course of the disease. The liver was enlarged $6 \mathrm{~cm}$. below the costal margin, and the spleen was palpable in the size of about a fist; the superficial lymphatic glands were slightly enlarged. Small petechial hemorrhages on the skin of the neck, the trunk, the arms, the legs and the scrotum. Anemic murmurs at the heart apex and venoushum was heard. The urine: Acid ; free from albumin and sugar, and much urobilinogen; negative to diazo reaction. The stool : negative to benzidine test and no egg of ascaris (but later a few eggs of anchylostomum duodenale were identified). Blood serum gave the negative Wassermann and Murata reactions. The Manto ux (1:1000 and 1:100) and Pirquet reactions showed negative results. The ophthalmoscopic findings*: Perivascular retinal hemorrhages and infiltrations over the fundus of the right eye, presenting features as in the case of retinitis leucaemica.

Blood picture was as follows:- Hemoglobin 25 (Sahli's hemoglobinometer value); red cells 1,400,000; colour index 1.1; platelets 280,000 by Lampert's ${ }^{6)}$ thrombocyte counting method; total white cells 12,000 ; lymphocytes $10,800(90 \%)$; neutrophiles $910(7.6 \%)$; eosinophiles $84(0.7 \%)$; basophiles $24(0.2 \%)$ and monocytes $180(1.5 \%)$. Neutrophile myelocytes occurred in only $0.2 \%$ (absolute count 24 per $\mathrm{cmm}$.), but none of more immature cells-no promyelocytes or myeloblasts. Slight aniso- and poikilocytosis, and polychromatophilia, but none of nucleated red cell (normoblast or megaloblast) was seen (Cf. Table 2, November 19). Coagulation (Biffi-Brooks' apparatus modified by $\left.\mathrm{S} \mathrm{a} \mathrm{to}^{7}\right)$ was completed at $71 / 2$ minutes, and bleeding time (D u ke's ${ }^{8)}$ method) slightly prolonged to 6 minutes.

* Here I wish to express my thanks to the Department of Ophthalmology of our University for the kind examination of the patient.

6) H. La m pert, Verh. dtseh. Ges. inn. Med., 1931, 43, 92.

7) K. Sa to, Ketsuekigaku no Kiso 1981, Tokyo 13.

8) W. W. Duke, Arch. of Intern. Med., 1912, 10, 445. 
The treatment in the hospital consisted almost entirely in repeated blood transfusions. As will be seen from Tảble 2, we repeated blood transfusions as often as 46 times during 5 months, and the total amount of blood transfused was 2060 c.c. An arsenic preparation (Liquor arsenic Fowleri), an iron preparation (ferrum reductum), cod liver oil, a liver preparation (hepan and hepatose) and copper sulphate were given with negative results. The detoxicating hormone of liver (yakriton $)^{9)}$ was used daily or every other day during the whole course of the disease. In February, carbon tetrachloride was used for anchylostomiasis 3 times. The liver and the spleen were enlarged throughout the whole course, though the size was often subject to a fluctuation. The treatment and the symptoms are shown in detail in Table 1 (Cf. Table 1).

At the end of December 1934, the general condition of the patient became very serious and the blood picture was as follows: Hemoglobin 25 (Sa hli's value), red cells $1,000,000$, platelets 150,000 , white cells 8,200 and neutrophiles 130 . In January 1935, the general condition improved, and at the end of that month, January 28 , red cell count reached $3,500,000$, platelets 360,000 and neutrophiles 4870 . The patient felt very well, appetite improved and petechial hemorrhages disappeared. But in February petechial hemorrhages occurred again all over the body. In March gingivitis and gangrenous stomatitis appeared, and foetor ex ore became more offensive. In April many nodular swellings on the skin of the head and the abdomen developed, and edema on the face was seen. From A pril 22 on, he suffered from oozing from the gum and the nose, and excreted black stools. He died on April 25, 1935, with the final diagnosis of aplastic anemia (Cf. Table 1).

\section{Blood exanination.}

Blood was examined as often as 31 times in the whole course of 5 months' duration. Leucocyte count was made by use of Sato and Shoji's ${ }^{10)}$ copper peroxidase method in the counting chamber and at the same time by use of Türk's solution. Blood films were stained with the Tohoku Pediatric Method, ${ }^{11)}$ a modification of Sato and Se-

9) A. S a to, Tohoku J. Exp. Med., 1926, 8, 232.

10) A. S ato and K. Shoj i, Journ. Labor. and Clin. Med., 1928, 13, 1058 ; Tohoku Igaku Zassi, 1929, 9, 262.

11) A. Sato, T. Suzuki and Ry. Shibata, Tohoku J. Exp. Med., 1934, 24, 195. 


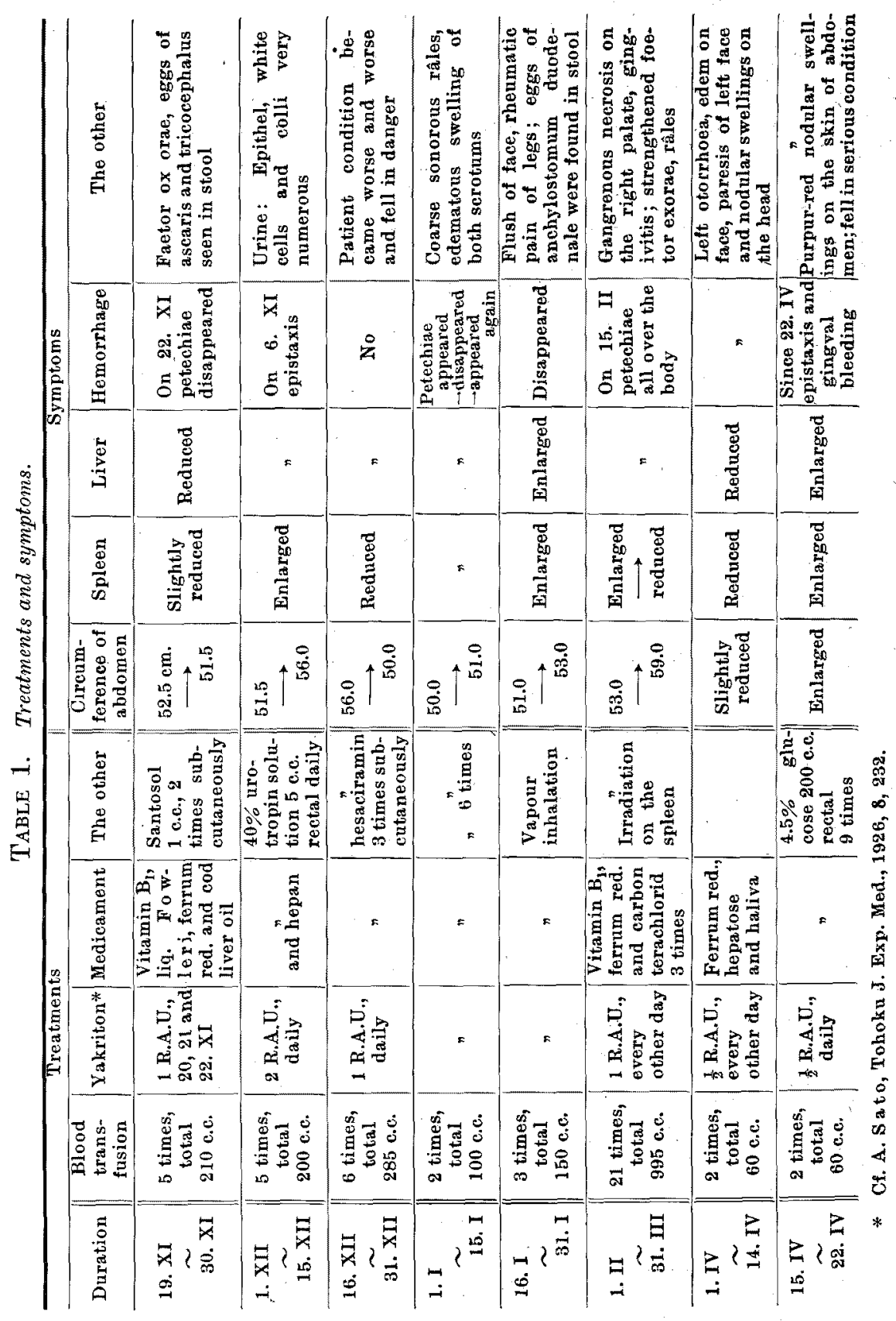


kiy a' ${ }^{12)}$ original copper peroxidase reaction.

The result of blood examination is given in Table 2. There was exceedingly remarkable anemia with slightly elevated colour index, slight aniso- and poikilocytosis, and polychromatophilia, but no basophilic stipplings, no nucleated red cells on the day of admission. A very few normoblasts occurred occasionally later.

1. Red cells: The red cell count was almost always below $2,000,000$, and the lowest fell to $1,000,000$. The count was over $2,000,000$ on December 4 and from the middle of January till the middle of February and at the end of March. At the other times it was below 2,000,000. On January 28 , the count reached 3,500,000 and the patient felt very well (Cf. Table 2 and Figure).

2. Hemoglobin: Hemoglobin fluctuated from 65 to 15 (Sahli's value), running almost parallel to the red cell count. The colour index was sometimes over 1 and sometimes below 1 (Cf. Table 2).

3. Blood platelets: The blood platelet count was within 420,000 and 90,000 by Lampert's ${ }^{6 /}$ thrombocyte counting method. Basing on my own result ${ }^{13)}$ for physiological platelet count, the count was almost always below the normal, except in the course from January to February when the patient's condition became better, and normal count was attained (Cf. Table 2 and Figure).

4. Pathological features in red cells : Slight aniso- and poikilocytosis existed during almost the whole course, but these disappeared before death. Very slight polychromatophilia occurred at the beginning of the disease and in January. Reticulocytes were examined only 4 times, showing a slight increase at each examination, the highest count being $3.4 \%$ on November 29 . A few normoblasts were occasionally seen, but megaloblasts never occurred (Cf. Table 2 ).

5. White cells : The normal count of white cells for the age of this patient is about 8,500 according Sa to-S uz u ki' ${ }^{14)}$ Standard Table for Every Age. In my own case the total leucocyte count was almost always below the normal limits except on these days : on the day of admission, on January 28, and on March 27. The lowest count was 900 and the highest was 12,000 (Cf. Table 2).

a. Lymphocytes: Lymphocytes showed generally a lymphopenia, but were over the normal limit* at the beginning and in Febru-

12) A. Sato and S. Sekiya, Tohoku J. Exp. Med., 1926, 7, 111.

13) Sh. Kimura, Tohoku J. Exp. Med., 1939, 37, 241.

14) T. Suzuki, Tohoku J. Exp. Med., 1937, 30, 378. 


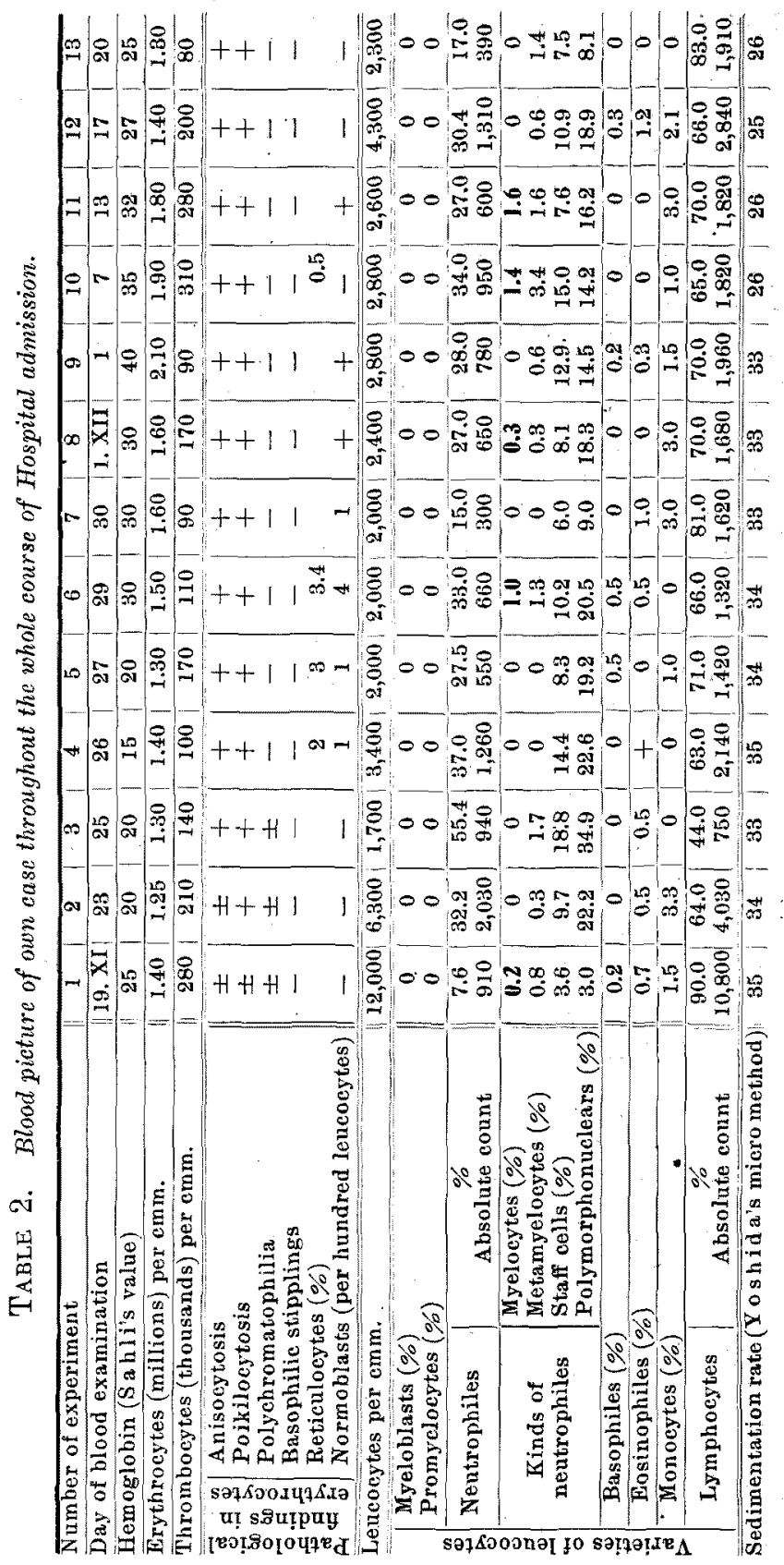




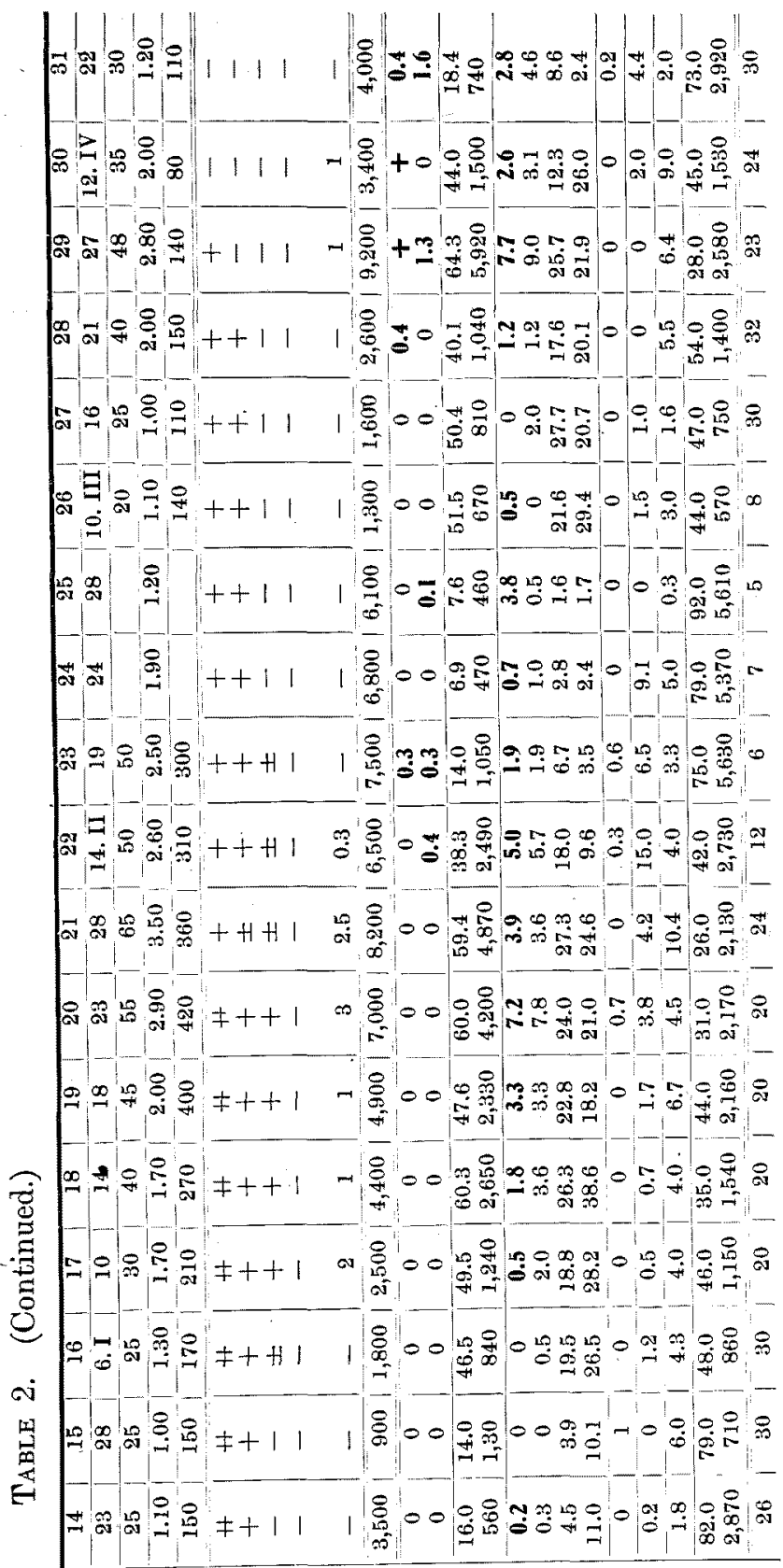


Figure. The fluctuation of erythrocytes, neutrophiles and thrombocytes throughout the whole course.

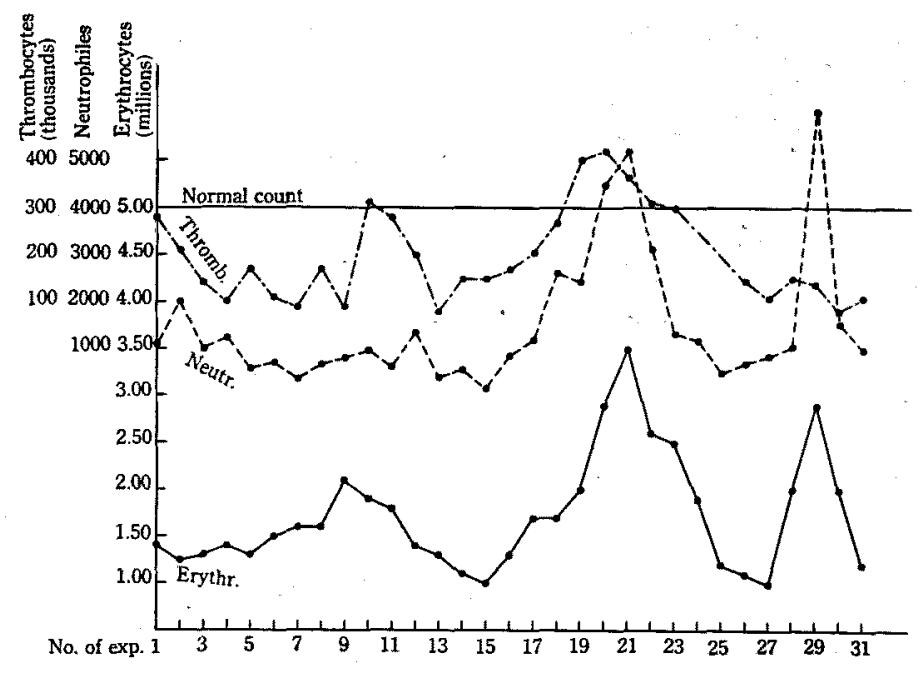

ary. During the whole course the lowest was 572 and the highest was 10,800 (Cf. Table 1). The great majority of lymphocytes were small ones.

b. Neutrophiles : Neutropenia was seen during almost the entire course except only 3 times : January 23 and 28, and March 27. The lowest was 130 and the highest was 5,850 (Cf. Table 2 and Figure). A rneth's nuclear shift was always inclined to the left, though in a typical case of aplastic anemia or panmyelophthisis a shift to the right is said to be the general rule.

c. Eosinophiles: Eosinophiles were below the normal limits relatively and absolutely through almost the whole course. Only 5 times (January 23, 28, February 14, 19 and 24) out of all the 31 times of blood examination, was that count over the normal limits. Sometimes a very few eosinophile myelocytes occurred (Cf. Table 2).

d. Basophiles: Basophiles occurred several times, but they were always below the normal limits (Cf. Table 2).

e. Monocytes: Monocytes were seen in almost the whole course, but always below the normal limits except twice (Cf. Table 2, Janu-

* Cf. Sat o-Suzuki's ${ }^{14}$ ) Standard Table. 
ary 28 and March 27 ).

f. Immature white cells: Myeloblasts and promyelocytes did not occur at all during the former three fifths of the whole course of the stay in hospital, and these immature cells were seen only occasionally (14. II, 19. II, 28. II, 21. III, 27. III and 22. IV) and quite a small number in the latter two fifths of the course. Myelocytes and metamyelocytes were also occasionally seen in a very small number (Cf. Table 2). It is to be noted here that, in such a case, if the nuclear shift of neutrophiles (including neutrophile metamyelocytes and myelocytes) is described by use of Arneth's method, one will notice the importance of the occurrence of myelocytes and metamyelocytes, even though these may in absolute count be small (Cf. Table 2 and see later).

\section{Post-mortem examination.*}

Generalized severe anemia of the whole body; grangrenous stomatitis ; marked edema of the larynx ; numerous petechial hemorrhages of duodenum and anchylostomiasis duodeuale; slight enlargement (the size of small finger tip) of superficial lymph nodes. Enlargement and metaplasia of myeloid cells of the kidneys, spleen, liver and mesenterial lymph nodes. Myeloid cell metaplasia of pleura, dura mater, periost of the skullbone, suprarenal capsule, endocard, gall bladder, testicle and nodular swellings of the skin. Intense myeloid metaplasia in lymph glands. Change of the kidneys was most interesting : 180 grms. (4 or 5 times the physiological weight for the age) ; the parenchyma could scarcely be seen on account of intensive metaplasia through myeloid cells. The bone marrow of the femur was remarkably soft and purple red with single purplebrown areas. Microscopically, the marrow was very cellular composed of myeloblasts, promyelocytes, myelocytes and more developed cells. Myeloblasts were relatively less numerous than the other more developed cells.

\section{Comment.}

In $1888 \mathrm{Eh} \mathrm{rli} \mathrm{h}{ }^{15}{ }^{15}$ describing a case of a young woman, 21 years old, who had developed an extremely severe anemia following inces-

* The writer acknowledges with thanks the assistance in this case from the Department of Pathology of our University.

15) P. Ehrlich, Charité-Annalen, 1888, 13, 300. 
sant uterine bleeding, proposed the name of 'aplastic anemia' because of a total absence of regenerative reaction on the part of the bone marrow. A number of papers have been published concerning this problem since Ehrlich's description. The most distinct feature of typical aplastic anemia is its unique blood picture. The red blood picture is of the non-regenerative type ; decided reduction in the total number of erythrocytes, hypochromia explaining in part the very low hemoglobin reading, anisocytosis, poikilocytosis and polychromatophilia are never encountered, nucleated red cells are never present and reticulocytes are very scarce. The bone marrow must be aplastic or fatty in a typical case. But actually there are many cases that are regarded as ones of aplastic anemia having severe anemia with a slight evidence of regenerative reaction of red blood picture and bone marrow. Thompson, Richter and $\mathrm{Edsall}{ }^{16)}$ found that 7 cases out of all the 13 cases of aplastic anemia analysed by themselves had the extremely cellular marrow with marked increase in one or more developing cell types. They observed also an occasional appearance of normoblasts as an evidence of erythrogenic activity and the shifting nucleus of neutrophiles to the left in all 13 cases.

The term ' aplastic anemia' is concerned only with erythrocytes and erythropoietic tissues, but the disease was often characterized with persistent leucopenia mainly due to neutropenia and with a conspicuous reduction in platelet count at the same time with an aplasia of red cells. A case in which the 3 symptoms above mentioned-anemia, neutropenia, and thrombocytopenia-occurred in a high grade and in combination was called panmyelophthisis or aleukia haemorrhagica (Frank ${ }^{17)}$ ).

The present case of mine consisted in a severe anemia with slight occurrence of regenerative reaction, in a considerable neutropenia mainly due to neutropenia and in a moderate thrombocytopenia. The case was thus regarded as an advanced stage of aplastic anemia or panmyelophthisis. The occasional occurrences of a few myelocytes and of a quite few promyelocytes and myeloblasts in the terminal stage were considered as a peculiar reaction of bone marrow irritation resulting from the repeated blood transfusions, until the post-mortem examination was performed.

The case that was in the early stage clinically similar to aplastic

16) W. P. Thompson, M. N. Richter and K. S. Edsall, Am. J. Med. Sc.. $1934,187,77$.

17) E. Fran k, Berl. klin. Wschr., 1915, 52, 951 and 1062. 
anemia or panmyelophthisis, and then proved from the blood picture in the terminal stage to be a well defined myeloid leukemic case was reported by Binder Lászl ${ }^{18)}$ and Penati ${ }^{19)}$ in recent years. Webe $r^{20)}$ reported a case of alenkemic myelosis under the heading 'aleukaemic myelosis of the leucopenic type, clinically simulating chronic aplastic anemia.' Weber and Weiss wange $\mathrm{e}^{21)}$ described the same case reported by Weber himself under the heading ' aplastische Anamie und Leukämie' and emphasized that behind an aplastic anemia, aleukemic forms of lymphadenosis and myelosis were often concealed. The case of Weber had a considerably numerous occurrence of myeloblasts in the latter half of the course, so that it was probably not very difficult to diagnose as a case of myelosis of leucopenic type without post-mortem examination. Segerdahl ${ }^{22)}$ had a case of leucopenia with marked anemia whose termination was an acute myelosis. The bone marrow of the patient was shown by autopsy one part to be atrophic and noncellular, and the other part to be an area of congregation of myeloblasts. Denecke $\mathrm{e}^{23)}$ reported a case who was early an aplastic anemia, and who later rapidly combined aleukemic lymphadenosis accompanied by greatly enlarged liver and spleen and swollen lymph nodes. Seiler and Heisler ${ }^{24}$ described a case of myeloid leukemia in early stage and who showed the terminal picture of aleukemia after a long course of the disease.

The case of mine was proved at autopsy to be a myeloid leukemic case whose clinical blood picture was nothing else than aplastic anemia or panmyelophthisis. A case similar to mine was reported in 1935 by Henschen and Jezler ${ }^{5\rangle}$ under the heading 'Aleukämische Myelose unter dem Bild der Panmyelophthise.' Their case was a typical panmyelophthisis as far as blood picture was concerned-no regenerative reaction of peripheral red cells, thrombocytopenia, and no myelocytes though the nuclear shift of neutrophiles was not to the right. This case was revealed to be a myelosis at the post-mortem examination. They said that such a case had not been published in literature until then, despite the fact that, in reality, the occurrence of such cases,

18) Binder Lászaló, Orvosi Hetilap, 1936, 80, 632.

19) F. Penati, Minerva Medica, 1937, 28, 627.

20) F. P. W e b er, Quart. Jour. Med., 1932, 25, 409.

21) F. P. We ber and W. Weis s ange, Dtsch. Arch. klin. Med., 1933-34, 176 $-422$.

22) E. Segerdah 1, Folia Haemat, 1934, 52, 68.

23) G. Denecke, Dtsch. Areh. klin. Med., 1926, 150, 266.

24) V. J. Seiler and W. Heisler, Münch. med. Wschr., 1935, 82, 577. 
they stated, was not rare. Their case had a remarkable megalosplenia and splenectomy was performed. Hicklin $\mathrm{g}^{25)}$ used for convenience" sake the term 'chronic non-leukaemic myelosis' in his paper to report on the patient who presented massive enlargement of the spleen due to myeloid metaplasia, and in whom the characteristic blood picture of leukemic myelosis was not found. Such a case named ' chronic nonleukaemic myelosis' by Hickling was of the same nature as the cases under the term of 'aleukemic' or 'sub-leukemic' myelosis described by several authors. So the case of Henschen and Jezler would be one belonging to chronic non-leukemic myelosis.

Aleukemic or leucopenic myelosis may clinically simulate aplastic anemia when the red blood picture is of the non-regenerative type. However the blood picture of the present case may resemble that of aplastic anemia or panmyelophthisis, we must not overlook the following conditions, though these occur very slightly:-Slight regenerative signs of red cells, and an occurrence of myelocytes, especially temporary occurrence of a very few myeloblasts and promyelocytes in the terminal stage. So the picture is not a typical one for aplastic anemia. Moreover, by a careful observation of clinical features throughout the whole course, one would notice an unnatural syndrome for aplastic anemia :-Massive enlargement of the liver and the spleen; the change of retina showing the picture of retinitis leucaemica and the nodular swelling of the skin of the abdomen and the head. The above described symptoms are a frequent syndrome seen in a case of myeloid leukemia. Had I paid special attention to these items at the clinical examination I might have been able to diagnose the present case correctly.

As described at the beginning of the present article, if one takes into consideration the possibly leukemic changes of blood forming organs (reported in literature) in apparent cases of aplastic anemia, and then pays special attention to the (even slightest) typical qualitative alteration of the peripheral blood picture, the exact diagnosis of aleukemia can be established without much difficulty. But it is very difficult to make correct diagnosis in such a case where there is no myelocyte and no myeloblast in the streaming blood, almost just as in the present case (Cf. blood picture on November 23, 25, 26, 27, 30 December $4,17,20,28$, January 6 and March 16 in Table 2). In such a case the attention must be directed to the clinical features and the slight-

25) R. A. Hickling, Quart. Jour. Med., 1937, 30, 253. 
est alteration of peripheral blood picture. And yet the exact diagnosis would often be made possible only after the post-mortem examination.

I emphasize again as follows :- If in an apparent case of aplastic anemia the blood picture is not entirely typical, and if there is, excepting the blood picture, the clinical feature which may be considered as a feature of leukemia, we must think of the possible diagnosis of aleukemic leukemia.

The present case was indubitably one of myeloid aleukemia revealed by pathologic-histologic examination, resembling a case of aplastic anemia or panmyelophthisis in the blood picture.

In connexion with the present case I should like to quote one of Rosenthal's ${ }^{26)}$ conclusions regarding the difference of aplastic anemia and agranulocytosis from leucopenic myelosis:- 'Leucopenic myeloid leukaemia may symptomatically resemble some of these blood conditions, but the constant presence of the characteristic myeloblasts and myelocytes or lymphoblasts in the peripheral blood is important for the differentiation of this particular disease.'

The present case is an example of aleukemic myelosis under the guise of aplastic anemia.

Aleukemic myelosis is the term used to describe a type of leukemia in which there is leukemic infiltration of many organs and in which the total white cell count of the peripheral blood is yet within normal limits or even leucopenic. Aleukemic myelosis has two clinical pictures in qualitative blood ohange. One of them is that the qualitative alteration of the peripheral blood is the same as seen in a leukemic case, only without a marked leucocytosis. The other is that the differential count of peripheral blood is apparently normal. The former case is quite the same as that of typical myeloid leukemia except for the total white cell count, so the diagnosis is not difficult. The diagnosis of the latter case is very difficult; it can be made only by postmortem examination. The present case is an example of the latter case with the clinical feature resembling aplastic anemia. But in such a case, if we observe most carefully the clinical feature and never overlook the slightest qualitative alteration of the blood picture, we shall be able to diagnose it as one of aleukemic myelosis without autopsy. Even the slightest qualitative neutrophile alteration of the peripheral blood according to Schilling's method in such a case would-so to

26) N. Rosenthal, Am. J. Clin. Path., 1931, 1, 7. (Cited from F. P. Weber, Quart. Jour. Med., 1932, 25, 409.) 
speak-be exaggerated if shown by the Arneth method of nuclear shift and induce one to pay special attention to the importance of neutrophile blood picture. For instance, take the picture seen on February 28, when myelocytes occurred in the largest percentage by Arneth's shift; the distribution of neutrophiles on February 28: Myelocytes $3.8 \%$, metamyelocytes $0.5 \%$, staff cells (or rhabdocytes) $1.6 \%$ and polymorphonuclears $1.7 \%$ by Schilling's shift was changed to the following: Myelocytes 50\%, metamyelocytes 7\%, staff cells 21\% and polynuclears $22 \%$ by A rneth's shift. If one looks over the picture showed by the Arneth nuclear shift, one may at once notice the very abnormally abundant occurrence of myelocytes, and moreover be led to diagnose the disease decidedly as an aleukemic myelosis.

I here emphasize that if one sees a typical aplastic picture one must most carefully observe the clinical feature and the finest qualitative alteration of the peripheral blood picture. And I also propose that nuclear picture of neutrophile leucocytes must be examined by use of the Arneth nuclear shift in the case of aplastic anemia. Then, almost all the aleukemic myelosis will be exactly diagnosed without autopsy, however strong the peripheral blood picture shows similarity to aplastic anemia or panmyelophthisis.

\section{Summary and Conclusions.}

1. In the present paper a case of aleukemic myelosis under the clinical guise of aplastic anemia was reported.

2. Behind apparent aplastic anemia there remains often an aleukemic myelosis. So if myelocytes happen to occur during repeated blood examinations, the clinical diagnosis: "Aplastic anemia " must not always be considered as faultless.

3. In such a case, the Arneth nuclear shift is to be noted besides the Schilling hemogram, so that one may not overlook the slight but important qualitative neutrophile alteration of streaming blood picture.

4. In a case of apparent aplastic anemia one must always consider the possible diagnosis of aleukemic myelosis and pay special attention to the neutrophile blood picture, especially if the clinical course presents a feature similar to that of leukemia. 